\title{
Layer-by-Layer Assembly of Cationic Guar Gum, Cellulose Nanocrystals and Hydroxypropyl Methylcellulose based Multilayered Composite Films
}

\section{Arun Saini}

Shaanxi University of Science and Technology Xil'an Campus: Shaanxi University of Science and Technology

\section{Deepak Sharma}

Forest Research Institute Dehradun

\section{Yuanyuan Xia}

Shaanxi University of Science and Technology Xi\'an Campus: Shaanxi University of Science and Technology

\section{Aman Saini}

Chaudhary Charan Singh University

\section{Xiangyu You}

Shaanxi University of Science and Technology Xi'an Campus: Shaanxi University of Science and Technology

\section{Ying Su}

Shaanxi University of Science and Technology Xi'an Campus: Shaanxi University of Science and Technology

\section{Lihong Chen}

Shaanxi University of Science and Technology Xi'an Campus: Shaanxi University of Science and Technology

Chandravati Yadav ( $D$ 4495@sust.edu.cn)

Shaanxi University of Science and Technology Xi'an Campus: Shaanxi University of Science and Technology https://orcid.org/0000-0002-9767-5244

\section{Xinping Li}

Shaanxi University of Science and Technology Xi'an Campus: Shaanxi University of Science and Technology

\section{Research Article}

Keywords: Carboxylated cellulose nanocrystals, multilayered composite film, flexible and strong, transparent, gas and oil barrier 
Posted Date: February 24th, 2021

DOl: https://doi.org/10.21203/rs.3.rs-230907/v1

License: (9) This work is licensed under a Creative Commons Attribution 4.0 International License. Read Full License

Version of Record: A version of this preprint was published at Cellulose on July 21 st, 2021 . See the published version at https://doi.org/10.1007/s10570-021-04064-6. 


\section{Abstract}

Eco-friendly sustainable materials provide an appealing template to replace contemporary syntheticnonrenewable resource-based materials while maintaining the acceptable material properties to meet the performance requirements. Here, a layer-by-layer ( $L B L)$ self-assembly technique was used for fabricating multilayer composite films using all bio-based polymers/polysaccharides, i.e. cationic guar gum (CGg), carboxylated cellulose nanocrystals (cCNCs) and hydroxypropyl methylcellulose (HPMC). A five layered composite film was fabricated by depositing polymeric layers as follows:

$\mathrm{CGg} \rightarrow \mathrm{cCNCs} \rightarrow \mathrm{HPMC} \rightarrow \mathrm{cCNCs} \rightarrow \mathrm{CGg}$. The structural analysis of $\mathrm{CGg} / \mathrm{cCNCs} / \mathrm{HPMC}$ multilayered composite films indicated the existence of electrostatic interaction as well as $\mathrm{H}$-bonding between polymeric layers that resulted in homogenous, dense and compact film surface with improved smoothness and strength properties. As compared to pure CGg film, the CGg/cCNCs/HPMC multilayered composite films showed improved tensile strength (84.8\% increment) and modulus (29.19\% improvement). Importantly, the deposition of HPMC layer contributed in achieving multilayer composite films with more flexible behavior (46.55\% improvement in elongation at break). Furthermore, owing to the high transparency (89.5\% transmittance), appreciable gas and oil barrier performance and resistance to various solvents (e.g. acetone, THF and DMAc), these multilayer films are promising candidates for various applications including renewable/sustainable packaging materials.

\section{Introduction}

The global demand of safe, ecofriendly, biodegradable and sustainable packaging materials containing quality products have compelled the development of novel packaging solutions (Anukiruthika et al. 2020, Sanchez-Garcia et al. 2010). In this context with the aim of developing novel packaging materials with improved performance, fabrication of multilayered composite films that integrates the unique functionalities of different polymers is emerging (Anukiruthika et al. 2020). In general, customary packaging materials are derived from non-sustainable synthetic polymers like polyethylene (PE), polypropylene (PP), and polystyrene (PS) due to their relatively low cost, ease of processing and excellent mechanical and barrier properties (Anukiruthika et al. 2020, Sanchez-Garcia et al. 2010). However, noneconomical plastic recycling processes and non-biodegradability of these petrochemical based plastics pose serious environmental issues. Hence, developing biodegradable packaging materials from biodegradable plastics (biopolymers) and resources is among the judicious strategies to mitigate or reduce the environmental impact of synthetic plastics.

Among various biopolymers, polysaccharides such as starch, chitosan, carrageenan, and galactomannans are most widely studied for the preparation of edible or biodegradable films. Guar gum $(\mathrm{Gg})$, a heteropolysaccharide/galactomannan is the powdered endosperm derived from the seeds of Cyamopsis tetragonolobus. Structurally, it possesses a straight chain of mannose linked through a $(1 \rightarrow 4)-\beta$-D-mannopyranose (Man) units and having branched galactose units linked through $(1 \rightarrow 6)$-a-Dgalactopyranose (Gal) units (Chudzikowski, 1971, Sharma et al. 2020) which is responsible for its thickening, emulsifying, binding, gelling and film forming properties in aqueous solutions (Thombare et 
al. 2016). However, Gg films exhibit relatively poor mechanical and barrier properties (Dai et al. 2017, Saurabh et al. 2015). Because of the moderately poor mechanical and rigidity properties of $\mathrm{Gg}$, there is an incredible inspiration to improve the physicochemical properties of the $\mathrm{Gg}$ based films by amalgamating them with other biopolymers. The last decade signifies the tremendous interest in the use of cellulose nanomaterials, like cellulose nanocrystals (CNCs), as bio-based nano-reinforcement for improving the mechanical properties of various polymeric materials (Bilbao-Sainz et al., 2011, Nair et al., 2014, Yadav et al., 2018, Yadav et al., 2020). Furthermore, the rod-like, highly crystalline CNCs can form dense H-bonded network structure that results in the formation of a tortuous path restricting the diffusion of aroma, taint, or other gases, thereby presenting excellent barrier properties suitable for packaging applications (BilbaoSainz et al., 2011, Nair et al., 2014).

The LBL self-assembly technique introduced by Decher, Hong, and Schmitt (1992) for the functionalization of a solid surface has become one of the most prominently utilized process for the fabrication of multilayered composite films (Decher et al. 1991). The technique is based on the alternate adsorption of components, like a polymer, biomolecule, or inorganic particle, onto a substrate that allows the sequential formation of a nanostructured composite film (Ding et al., 2005; Costa et al., 2013). The fabrication of multilayered composite films through LBL self-assembly technique usually involves the deposition of oppositely charged polyelectrolytes via ionic interactions (Costa et al., 2013). However, LBL construction mechanism can be driven by several other interactions, including electrostatic interactions, $\mathrm{H}$-bonds, charge transfer interactions, guest-host interactions, cation-dipole interactions, or the combined interactions of the above forces (Li, et al., 2012; Zhu et al., 2015). LBL technique offers advantage of adequate interfacial interaction by achieving proper dispersibility or miscibility of various polymers used (Sui et al., 2010). Hence, the multilayered functional composite films with well-defined thickness, composition, and structure can be easily fabricated from the aqueous solutions (Decher, 1997). The simple easy-to-apply methodology, flexibility in the use of materials with the possibility of using different active constituents and thickness control ability at the nanoscale level perhaps make LBL selfassembly technique a versatile surface-modification tool (Qi et al., 2012; Castleberry et al., 2014). The technique offers advantage of imparting unique physicochemical properties for preparing smart composite film surfaces and advanced coatings for various applications (Castleberry et al., 2014; Richardson et al., 2015). Such LBL structured composite films have a wide scope of utilization in packaging materials, electrochromic devices, optical sensors, super-hydrophobic surfaces, dye-sensitized solar cells, tissue engineering etc. (Lee et al. 2000; Ogawa et al. 2007; Kokubo et al. 2007, Richardson et al. 2015).

LBL self-assembly approach can comprise various approaches like spraying, immersion, spinning and casting (Richardson et al. 2015). Various research groups have reported the use of LBL technique for fabricating multilayered composites by combining anionic CNCs with various cationic polymers like poly (diallyldimethylammonium chloride) (Pillai et al. 2016), poly(allylamine) hydrochloride (Moreau et al. 2012), poly(ethylenimine) (Li et al. 2019) etc. As yet, very few research groups have reported the fabrication of $\mathrm{Gg}$ based multilayered composites using LBL technique. Dai et al. (2017) developed composite films by depositing alternating layers of CGg and anionic CNFs to fabricate robust 
multilayered films with good oil and gas barrier properties. In the present work, cationic guar gum (CGg), carboxylated cellulose nanocrystals (cCNCs) and hydroxypropylmethyl cellulose (HPMC) were used to fabricate multilayered composite films by employing LBL self-assembly technique. HPMC, a cellulose ether with excellent film forming properties, has been used here as a flexible interface in order to generate flexible transparent films with good optical, mechanical and barrier properties. The main objective of the presented work is to develop bio-based, sustainable and environmentally benign multilayered composite films with intended application as packaging materials.

\section{Experimental Section \\ 2.1. Materials}

The bleached bamboo pulp obtained from Guizhou Chitianhua Co., Ltd. was used as the raw material for the preparation of carboxylated cellulose nanocrystals (cCNCs). Sodium chlorite, acetic acid, citric acid and sulfuric acid were purchased from Tianjin DaMao Chemical Reagent Factory, Tianjin, China. Cationic guar gum (CGg) was kindly provided by Yan Cheng Xin Yuen Chemical Co. (Yancheng, China). Potassium hydroxide and hydroxypropyl methylcellulose (HPMC) ( $M_{n}: 22,000$; Methyl substitution: 28-30\%; Hydroxypropyl substitution: 7-12\%) was procured from Aladdin Chemistry Co., Ltd., China. All the chemicals and reagents were used as received without any further purification.

\subsection{Preparation of $\mathrm{CGg} / \mathrm{cCNCs} / \mathrm{HPMC}$ multilayered composite film}

The simultaneous production and carboxylation of cellulose nanocrystals (cCNCs) from purified cellulose fibres was carried out following our previously reported method (Saini et al. 2019). Briefly, a mixed acid assisted hydrolysis system containing a mixture of a strong acid $\left(\mathrm{H}_{2} \mathrm{SO}_{4}, 20 \mathrm{wt} \%\right.$ ) and a weak acid (citric acid, $40 \mathrm{wt} \%)$ under hydrothermal reaction conditions $\left(4 \mathrm{~h}, 100^{\circ} \mathrm{C}\right)$ was adopted. The detailed reaction scheme has been shown in Fig. S1 (Supporting information). The LBL self-assembly technique was used to prepare $\mathrm{CGg} / \mathrm{cCNCs} / \mathrm{HPMC}$ multilayered composite film. CGg and HPMC powder were dissolved separately in deionized water under slow stirring to prepare a homogenous solution of $0.5 \mathrm{wt} \%$ and 0.1 wt\%, respectively. $20 \mathrm{~g}$ of prepared CGg solution was poured in a Petri dish as the first layer and dried in hot air oven at $50^{\circ} \mathrm{C}$. Afterwards, $20 \mathrm{~g}$ of $0.5 \mathrm{wt} \%$ (optimized concentration) cCNCs suspension was poured on the top of the partially dried/moist CGg layer. $20 \mathrm{~g}$ of $0.1 \mathrm{wt} \% \mathrm{HPMC}$ solution was then dispensed over moist cCNCs layer. The process was repeated in following pattern:

$\mathrm{CGg} \rightarrow \mathrm{cCNCs} \rightarrow \mathrm{HPMC} \rightarrow \mathrm{cCNCs} \rightarrow \mathrm{CGg}$. The deposited layers were then fully dried at $50^{\circ} \mathrm{C}$ in hot air oven for fabricating a five layered $\mathrm{CGg} / \mathrm{cCNCs} / \mathrm{HPMC}$ multilayered composite films. The same process was followed for the preparation of $\mathrm{CGg} / \mathrm{cCNCs}$ multilayered (five layered) composite film. The pure CGg film $(0.5 \mathrm{wt} \%, 100 \mathrm{~g})$ was also fabricated to examine the effect of cCNCs and HPMC in the characteristics of prepared multilayered composite films. After drying at $50^{\circ} \mathrm{C}$, the prepared films were peeled off from Petri dishes. The prepared films were then placed at $60^{\circ} \mathrm{C}$ under vacuum for $24 \mathrm{~h}$ to remove any traces of bound moisture. The films were placed at $23^{\circ} \mathrm{C}$ and $50 \% \mathrm{RH}$ for at least 2 days before further 
characterization. The whole procedure of multilayered composite film fabrication using LBL technique has been shown pictorially in Fig. 1.

\subsection{Characterization}

The characterization of cCNCs has been detailed in our previously published report (Saini et al. 2019). The surface morphology examination results attained through transmission electron microscopy (TEM, FEI Tecnai G2 F20 TEM, USA) and atomic force microscopy (AFM, Dimension Icon, Bruker, USA) have been provided in Fig. S2 (Supporting information). The carboxylate content of cCNCs was $1.18 \mathrm{mmol} / \mathrm{g}$. The microstructural studies revealed that the length of nanocrystals lies in the range of $280-420 \mathrm{~nm}$ with diameter ranging from 10 to $20 \mathrm{~nm}$ (Saini et al. 2019).

Tensile properties of films (rectangular strips: $70 \mathrm{~mm}$ long, $10 \mathrm{~mm}$ wide) were tested on GOTECH UTMuniversal testing machine. The distance between grips (gauge length) was maintained as $50 \mathrm{~mm}$. The testing was performed at room temperature with a cross head speed of $10 \mathrm{~mm} / \mathrm{min}$ and load cell of 500 $\mathrm{N}$. Five specimens of each sample were tested, and the values obtained were averaged to report the results.

The optical properties of films were analyzed using ultraviolet - visible spectrophotometer (UV - vis, Shimadzu UV-2550) in the wavelength region of 200 to $900 \mathrm{~nm}$.

Morphological study of films was carried out through Field emission scanning electron microscopy (FESEM, S4800, Hitachi, Japan). For cross-section analysis the films were fractured in liquid nitrogen. The gold coated surfaces were then mounted on aluminum stubs and images were captured with an accelerating voltage of $10 \mathrm{kV}$ at a working distance of $5 \mathrm{~mm}$.

Fourier transform infrared spectroscopy (FTIR, Bruker Vertex 70, Billerica, USA) was used for chemical interaction analysis of prepared films in attenuated total reflectance (ATR) mode (PIKE MIRacle ATR accessory, Madison, WI). All the spectra were recorded in the spectral range of $4000-600 \mathrm{~cm}^{-1}$ at a resolution of $4 \mathrm{~cm}^{-1}$ and accumulation of 32 scans for each sample.

Solvent resistance and absorption study of multilayered composite film was investigated by immersion of $4 \mathrm{~cm} \times 1 \mathrm{~cm}$ strips in various solvents (water, acetone, THF and DMAc) for $48 \mathrm{~h}$. To determine the solvent uptake percentage, the samples were removed from the solvents at specific time intervals $(0 \mathrm{~h}, 12$ h, $24 \mathrm{~h}$ and $48 \mathrm{~h}$ ), gently wiped with filter paper to remove excess solvent from surface and were weighed. The following equation was used to measure the solvent uptake (\%):

$$
\text { Solvent uptake }(\%)=\left[\frac{w_{t}-w_{o}}{w_{o}}\right] \times 100 \%
$$

where, $w_{t}$ is the film weight after being immersed in solvent for a certain time and $w_{o}$ is the initial film weight. 
The water vapor transmission rate (WVTR) of multilayered composite films was measured using water vapor permeability tester (PERME TSY-TIL, Labthink Instruments Co., Ltd, Jinan, China) at RT and 90\% relative humidity $(\mathrm{RH})$.

Oil resistance test of multilayered composite film was performed using mustered oil (edible oil). A blank filter paper (stain absorber) was placed in a Petri dish. The testing film was immersed in oil and was immediately placed over the blank filter paper. After some time, the film was taken off from Petri dish and was wiped with tissue paper to observe the stained spots over the surface of tested film.

\section{Results And Discussion}

The packaging materials should possess good mechanical attributes in order to resist external impact and to maintain the physical integrity of the packaged material. The compatible interaction between various polymers ensures the improved mechanical strength of a composite film (Dai et al. 2015; Cheng et al. 2016). The tensile stress-strain curve shown in Fig. 2 demonstrates the influence of CGg, cCNCs and HPMC polymeric layers on the mechanical properties of fabricated multilayered composite film. A significant improvement in tensile strength (from $17.83 \mathrm{MPa}$ to $32.44 \mathrm{MPa}$ ) and modulus (1.61 GPa to $2.83 \mathrm{GPa}$ ) was noted with the deposition of cCNCs layer on CGg layer as compared to pristine CGg film. Such improvement can be attributed to the compatible and good H-bond interaction between CGg and cCNCs that could have led to the formation of a resistant network structure, thereby resulting in effective stress transfer at the CGg-cCNCs interface (Cheng et al. 2016; Saini et al. 2019). Moreover, mechanical properties can also get benefitted from the electrostatic interactions between carboxyl groups of cCNCs and quaternary ammonium groups of $\mathrm{CGg}$ (Dai et al. 2017). However, a decrease in the percent elongation at break (from $2.32 \%$ to $1.80 \%$ ) was observed with the incorporation of cCNCs layer in $\mathrm{CGg} / \mathrm{cCNCs}$ multilayered composite film. The most probable reason for this trend can be accredited to the inherent rigid/stiff nature of cCNCs that restricted the motion of polymer matrix (Cheng et al. 2016; Dai et al., 2015). From the social and economic trends observed in last decade it is evident that flexible packaging formats are gradually replacing the rigid pack formats due to reduced material use and packaging/transport

costs (Marsh et al. 2007). Hence, to generate flexible composite films, HPMC polymeric layer was introduced. As is apparent from the stress-strain curve (Fig. 2a), the elongation at break increased from $1.80 \%$ for $\mathrm{CGg} / \mathrm{cCNCs}$ to $3.40 \%$ for $\mathrm{CGg} / \mathrm{cCNCs} / \mathrm{HPMC}$ multilayered composite film. The increase in elongation can be due to the weakening of interaction forces between CGg-cCNCs chains that escalated the polymer chain mobility under stress due to increased intermolecular spacing. At the same time, the generation of new $\mathrm{H}$-bonds contributed towards achieving appreciable strength (32.95 $\mathrm{MPa}$ ) and modulus (2.08 GPa) properties for $\mathrm{CGg} / \mathrm{cCNCs} / \mathrm{HPMC}$ multilayered composite film.

The optical properties of the composite film is directly related to its degree of homogeneity, compatibility between components and the internal microstructure (Cheng et al. 2016; Chu et al. 2020). The poor compatibility between two polymers typically results in reduced light transmittance of composite films 
due to the occurrence of light reflection or scattering at the phase interface (Chu et al. 2020). The flexible, smooth and transparent nature of $\mathrm{CGg} / \mathrm{cCNCs} / \mathrm{HPMC}$ multilayered composite film (Fig. 3 inset) depicted good compatibility between the polymers used for the film fabrication. In addition, the optical transparency of the prepared films was evaluated using UV - Vis spectrophotometer by acquiring light transmittance through the films as a function of wavelength (Fig. 3). The transmittance for pure CGg film,

$\mathrm{CGg} / \mathrm{cCNCs}$ and $\mathrm{CGg} / \mathrm{cCNCs} / \mathrm{HPMC}$ multilayered composite film at $600 \mathrm{~nm}$ was recorded around $85.4 \%$, $87.9 \%$ and $89.5 \%$, respectively. The higher transparency of $\mathrm{CGg} / \mathrm{cCNCs} / \mathrm{HPMC}$ multilayered composite film depicted the uniform deposition and good interaction of nanodimensional cCNCs layer with sequential layers of CGg and HPMC in five-layered composite film. The lower transmittance of pure CGg is probably due to the increased light scattering from larger polymer particles. While the lower transparency of $\mathrm{CGg} / \mathrm{cCNCs}$ multilayered composite film can be related to the presence of some clustered cCNCs responsible for light scattering from the film surface. These observations revealed the efficient role of HPMC in improving the film forming abilities by enabling better polymeric interactions that can be well corroborated with the mechanical properties results.

The microstructure of multilayered composite films can provide relevant insight about the structural compatibility between various polymers used for film fabrication. The cross-section image of the pristine CGg film revealed a smooth surface (Fig. 4a). The addition of cCNCs layer caused noticeable changes in the microstructure of multilayered composite films. A layered but comparatively rougher surface was apparent for $\mathrm{CGg} / \mathrm{cCNCs}$ film (Fig. 4b) while for $\mathrm{CGg} / \mathrm{cCNCs/HPMC} \mathrm{film,} \mathrm{a} \mathrm{uniform,} \mathrm{well} \mathrm{deposited} \mathrm{and}$ smooth layered microstructure was

observed (Fig. 4c). This indicated the appreciable role of HPMC in improving polymeric interactions and compatibility in generating good interfacial adhesion. This compatibility was likely to be based on improved electrostatic interactions and rich $\mathrm{H}$-bonding between the polymeric layers. Overall, the high level of miscibility between the polymeric layers resulted in homogenous, dense and compact film surface with improved smoothness, flexibility, optical and mechanical performance of $\mathrm{CGg} / \mathrm{cCNCs} / \mathrm{HPMC}$ multilayered composite film.

FTIR-ATR was performed to analyze the effect of intermolecular interactions on the vibration of functional groups present in various polymeric components used for the fabrication of multilayered composite film. For all prepared films, the broad band in the region $3500-3000 \mathrm{~cm}^{-1}$ corresponds to $\mathrm{O}-\mathrm{H}$ stretching vibrations. The bands in the region $2980-2800 \mathrm{~cm}^{-1}$ represents the symmetric and asymmetric $\mathrm{C}-\mathrm{H}$ stretching vibrations of the aliphatic moieties. The shifting of absorption bands of $-\mathrm{OH}$ to lower wavenumber indicates the occurrence or strengthening of $\mathrm{H}$-bonding between polymeric components (Dong-Bao et al. 2004). For pristine CGg film, the band due to -OH with maximum intensity at around $3360 \mathrm{~cm}^{-1}$ shifted to around $3340 \mathrm{~cm}^{-1}$ for $\mathrm{CGg} / \mathrm{cCNCs}$ and $\mathrm{CGg} / \mathrm{cCNCs} / \mathrm{HPMC}$ multilayers composite films, respectively (Fig. 5). These changes in the $-\mathrm{OH}$ band position depicts the formation of $\mathrm{H}$ bonding that strengthens with the incorporation of HPMC layer in composite film. Further, the CGg spectra showed sharp bands at $1653 \mathrm{~cm}^{-1}$ and $1445 \mathrm{~cm}^{-1}$ depicting the presence of quaternary 
ammonium cations (Dai et al. 2020; Kaur et al. 2018). In case of CGg/cCNCs and CGg/cCNCs/HPMC multilayered composite film the characteristic band appeared at around $1635 \mathrm{~cm}^{-1}$ which can be related to the electrostatic interactions between carboxylate groups of cCNCS and quaternary ammonium cations of CGg and the overlapping of characteristic bands of CGg and cCNCs (Dai et al. 2020; Kaur et al. 2018). Further the band at $1445 \mathrm{~cm}^{-1}$ shifted to lower wavenumber at $1425 \mathrm{~cm}^{-1}$ for CGg/cCNCs film, suggesting the strong electrostatic interaction or H-bonding between CGg and cCNCs (Dai et al. 2017; Kaur et al. 2018). For CGg/cCNCs/HPMC multilayered composite film, the splitting of the $1445 \mathrm{~cm}^{-1}$ band was apparent at $1440 \mathrm{~cm}^{-1}$ and $1415 \mathrm{~cm}^{-1}$. This splitting may arise from the resonance interaction or coupling of vibration modes between analogous groups along the polymer chain (Mallapargada et al. 2006; Bao et al. 2010). The bands at $1380 \mathrm{~cm}^{-1}$ and $875 \mathrm{~cm}^{-1}$ depicting the $\mathrm{C}-\mathrm{N}$ stretching vibration and skeletal stretching vibration of pristine CGg film (Dai et al. 2017; Dai et al. 2019), respectively, were also evident in multilayered composite films with slight band shift. These revelations from FTIR-ATR spectra clearly indicates the strong interaction of $\mathrm{CGg}$ with both $\mathrm{CCNCs}$ and HPMC that may ultimately contribute towards the improved physical performance of multilayered composite films.

The solvent resistance or swelling properties of the $\mathrm{CGg} / \mathrm{cCNCs}$ /HPMC multilayered composite film was examined using various solvents like water, acetone, THF and DMAc. After $12 \mathrm{~h}$, the film sample in water started to swell but maintained its initial shape even after $48 \mathrm{~h}$ of immersion (Fig. 6). No visible variation in the film appearance was apparent for the films kept immersed for $48 \mathrm{~h}$ in other organic polar solvents (Fig. 6). Table S1 (Supporting Information) represents the quantitatively determined values for the solvent uptake or absorption by the film. The results attained clearly demonstrate the remarkable solvent resistance performance of the films against organic polar solvents like acetone, THF and DMAc while the hydrophilic nature of CGg, cCNCs and HPMC was probably the reason for the higher water absorption level of the $\mathrm{CGg} / \mathrm{cCNCs} / \mathrm{HPMC}$ multilayered composite film. Figure 7A depicts the WVTR of pristine CGg film, and $\mathrm{CGg} / \mathrm{cCNCs}$ and $\mathrm{CGg} / \mathrm{cCNCs} / \mathrm{HPMC}$ multilayered composite films. As compared to pristine $\mathrm{CGg}$, reduction in WVTR values for both $\mathrm{CGg} / \mathrm{cCNCs}$ and $\mathrm{CGg} / \mathrm{cCNCs} / \mathrm{HPMC}$ multilayered composite films was noticed. These improved barrier properties of multilayered

composite films can be attributed to the incorporation of highly crystalline cCNCs layer as well as increase in deposited layers that lowers the permeability by increasing the tortuosity thus leading to a slow diffusion process (De Moura 2011). Further, the enhanced barrier performance can also be a result of efficient interaction of composite components thereby generating a compact multilayered structure. Furthermore, the oil resistance property of multilayered composite films was also examined (Fig. 7B). The $\mathrm{CGg} / \mathrm{cCNCs} / \mathrm{HPMC}$ multilayered composite film that was kept immersed in oil, transferred all the oil to the filter paper (stain absorber) and

after wiping off showed a stain free or cleaner surface. This clearly indicates the oil repellent or oil impermeable nature of this polysaccharides based multilayered composite film. Typically, the high cohesive energy density of polysaccharide-based films plays a crucial role in generating excellent barrier properties against oil or grease (Wang et al. 2018, Senturk Parreidt 2018). Further, the compact structure 
of films and highly crystalline nature of cCNCs layer imparts major contribution towards the oil impermeant behavior of the fabricated composite film.

\section{Conclusion}

Multilayered composite films were fabricated by depositing alternate layers of CGg, cCNCs and HPMC through LBL self-assembly technique. The incorporation of cCNCs layer contributed in improving the strength properties while the HPMC polymeric layer attributed flexible attributes to the fabricated films. The high level of miscibility or compatibility was discernible from the uniform, well deposited and smooth layered microstructure of $\mathrm{CGg} / \mathrm{cCNCs} / \mathrm{HPMC}$ film that exhibited optimum optical and strength properties. Further, the fabricated films showed remarkable barrier properties against gas, oil and various solvents like acetone, THF and DMAc. Based on these findings and the renewable and biocompatible features the prepared composite films can have potential applications as green-sustainable packaging materials.

\section{Declarations}

\section{Acknowledgements}

This work was supported by National Natural Science Foundation of China (31370578) and Shaanxi Provincial Key Laboratory of Papermaking Technology and Specialty Paper Development, College of Bioresources Chemical and Materials Engineering, Shaanxi University of Science \& Technology.

\section{Declaration of competing interest}

The authors declare that they have no known competing financial interests or personal relationships that could have appeared to influence the work reported in this paper.

\section{References}

1. Anukiruthika T, Sethupathy P, Wilson A, Kashampur K, Moses JA, Anandharamakrishnan C (2020) Multilayer packaging: Advances in preparation techniques and emerging food applications. Compr Rev Food Sci Food Saf 19(3):1156-1186

2. Bao SP, Tjong SC, Tang CY (2010) Crystallization behavior of semicrystalline polymer-clay nanocomposites. In Phys Prop Appl Polym Nanocompos (pp. 280-314). Woodhead Publishing

3. Bilbao-Sainz C, Bras J, Williams T, Sénechal T, Orts W (2011) HPMC reinforced with different cellulose nano-particles. Carbohydr Polym 86(4):1549-1557

4. Castleberry SA, Li W, Deng D, Mayner S, Hammond PT (2014) Capillary flow layer-by-layer: a microfluidic platform for the high-throughput assembly and screening of nanolayered film libraries. ACS Nano 8(7):6580-6589

5. Cheng S, Zhang Y, Cha R, Yang J, Jiang X (2016) Water-soluble nanocrystalline cellulose films with highly transparent and oxygen barrier properties. Nanoscale 8(2):973-978 
6. Chu M, Feng N, An H, You G, Mo C, Zhong H, Hu D (2020) Design and validation of antibacterial and $\mathrm{pH}$ response of cationic guar gum film by combining hydroxyethyl cellulose and red cabbage pigment. Int J Biol Macromol 162:1311-1322

7. Costa RR, Testera AM, Arias FJ, Rodríguez-Cabello JC, Mano JF (2013) Layer-by-layer film growth using polysaccharides and recombinant polypeptides: a combinatorial approach. J Phys Chem B 117(22):6839-6848

8. Dai L, Cheng T, Wang Y, Lu H, Nie S, He H, Ni Y (2019) Injectable all-polysaccharide self-assembling hydrogel: a promising scaffold for localized therapeutic proteins. Cellulose 26(11):6891-6901

9. Dai L, Cheng T, Xi X, Nie S, Ke H, Liu Y, Chen Z (2020) A versatile TOCN/CGG self-assembling hydrogel for integrated wastewater treatment. Cellulose 27(2):915-925

10. Dai L, Long Z, Chen J, An X, Cheng D, Khan A, Ni Y (2017) Robust guar gum/cellulose nanofibrils multilayer films with good barrier properties. ACS Appl Mater Interfaces 9(6):5477-5485

11. Dai L, Wang B, Long Z, Chen L, Zhang D, Guo S (2015) Properties of hydroxypropyl guar/TEMPOoxidized cellulose nanofibrils composite films. Cellulose 22(5):3117-3126

12. De Moura MR, Avena-Bustillos RJ, McHugh TH, Wood DF, Otoni CG, Mattoso LH (2011) Miniaturization of cellulose fibers and effect of addition on the mechanical and barrier properties of hydroxypropyl methylcellulose films. J Food Eng 104(1):154-160

13. Decher G (1997) Fuzzy nanoassemblies: toward layered polymeric multicomposites. Science 277(5330):1232-1237

14. Decher G, Hong JD (1991, June) Buildup of ultrathin multilayer films by a self-assembly process, 1 consecutive adsorption of anionic and cationic bipolar amphiphiles on charged surfaces. In Makromolekulare Chemie. Macromolecular Symposia (Vol. 46, 1, pp 321-327). Basel: Hüthig \& Wepf Verlag

15. Ding B, Fujimoto K, Shiratori S (2005) Preparation and characterization of self-assembled polyelectrolyte multilayered films on electrospun nanofibers. Thin Solid Films 491(1-2):23-28

16. Dong-Bao HE, Li-hua L, Qing L, Xiao-zhen Y (2004) Synergistic interaction and gelation in cationic guar gum-sodium alginate system. Wuhan Univ J Nat Sci 9(3):371-374

17. Kaur J, Kaur G (2018) Optimization of pH conditions and characterization of polyelectrolyte complexes between gellan gum and cationic guar gum. Polym Adv Technol 29(12):3035-3048

18. Kokubo H, Ding B, Naka T, Tsuchihira H, Shiratori S (2007) Multi-core cable-like TiO2 nanofibrous membranes for dye-sensitized solar cells. Nanotechnology 18(16):165604

19. Lee SH, Kumar J, Tripathy SK (2000) Thin film optical sensors employing polyelectrolyte assembly. Langmuir 16:10482-10489

20. Li N, Zhang H, Xiao Y, Huang Y, Xu M, You D, Yu J (2019) Fabrication of cellulose-nanocrystal-based folate targeted nanomedicine via layer-by-layer assembly with lysosomal $\mathrm{pH}$-controlled drug release into the nucleus. Biomacromol 20(2):937-948 
21. Li Y, Wang X, Sun J (2012) Layer-by-layer assembly for rapid fabrication of thick polymeric films. Chem Soc Rev 41(18):5998-6009

22. Mallapragada SK, Narasimhan B (2006) Infrared spectroscopy in analysis of polymer crystallinity. Encyclopedia of analytical chemistry: applications, theory and instrumentation

23. Mamedov AA, Kotov NA (2000) Free-standing layer-by-layer assembled films of magnetite nanoparticles. Langmuir 16(13):5530-5533

24. Marsh K, Bugusu B (2007) Food packaging-roles, materials, and environmental issues. J Food Sci 72(3):R39-R55

25. Moreau C, Beury N, Delorme N, Cathala B (2012) Tuning the architecture of cellulose nanocrystalpoly (allylamine hydrochloride) multilayered thin films: influence of dipping parameters. Langmuir 28(28):10425-10436

26. Nair SS, Zhu J, Deng Y, Ragauskas AJ (2014) High performance green barriers based on nanocellulose. Sustain Chem Process 2(23):1-7

27. Ogawa T, Ding B, Sone Y, Shiratori S (2007) Super-hydrophobic surfaces of layer-by-layer structured film-coated electrospun nanofibrous membranes. Nanotechnology 18(16):165607

28. Pillai KV, Renneckar S (2016) Dynamic mechanical analysis of layer-by-layer cellulose nanocomposites. Ind Crops Prod 93:267-275

29. Qi ZD, Saito T, Fan Y, Isogai A (2012) Multifunctional coating films by layer-by-layer deposition of cellulose and chitin nanofibrils. Biomacromol 13(2):553-558

30. Richardson JJ, Björnmalm M, Caruso F (2015) Technology-driven layer-by-layer assembly of nanofilms. Science 348(6233)

31. Saini A, Yadav C, Xue BL, Wang N, Dai L, Li X (2019) Mixed-Acid-Assisted Hydrothermal Process for Simultaneous Preparation and Carboxylation of Needle-Shaped Cellulose Nanocrystals. ACS Appl Polym Mater 2(2):548-562

32. Sanchez-Garcia MD, Lopez-Rubio A, Lagaron JM (2010) Natural micro and nanobiocomposites with enhanced barrier properties and novel functionalities for food biopackaging applications. Trends Food Sci Technol 21(11):528-536

33. Saurabh CK, Gupta S, Bahadur J, Mazumder S, Variyar PS, Sharma A (2015) Mechanical and barrier properties of guar gum based nano-composite films. Carbohydr Polym 124:77-84

34. Senturk Parreidt T, Müller K, Schmid M (2018) Alginate-based edible films and coatings for food packaging applications. Foods 7(10):170

35. Sharma D, Kumar V, Sharma P (2020) Application, Synthesis, and Characterization of Cationic Galactomannan from Ruderal Species as a Wet Strength Additive and Flocculating Agent. ACS omega 5(39):25240-25252

36. Sui L, Huang L, Podsiadlo P, Kotov NA, Kieffer J (2010) Brillouin light scattering investigation of the mechanical properties of layer-by-layer assembled cellulose nanocrystal films. Macromolecules 43(22):9541-9548 
37. Thombare N, Jha U, Mishra S, Siddiqui MZ (2016) Guar gum as a promising starting material for diverse applications: A review. Int J Biol Macromol 88:361-372

38. Wang J, Gardner DJ, Stark NM, Bousfield DW, Tajvidi M, Cai Z (2018) Moisture and oxygen barrier properties of cellulose nanomaterial-based films. ACS Sustain Chem Eng 6(1):49-70

39. Yadav C, Saini A, Maji PK (2018) Cellulose nanofibres as biomaterial for nano-reinforcement of poly [styrene-(ethylene-co-butylene)-styrene] triblock copolymer. Cellulose 25(1):449-461

40. Yadav M, Behera K, Chang YH, Chiu FC (2020) Cellulose nanocrystal reinforced chitosan based uv barrier composite films for sustainable packaging. Polymers 12(1):202

41. Zhu Y, Xuan H, Ren J, Ge L (2015) Self-healing multilayer polyelectrolyte composite film with chitosan and poly (acrylic acid). Soft Matter 11(43):8452-8459

\section{Figures}

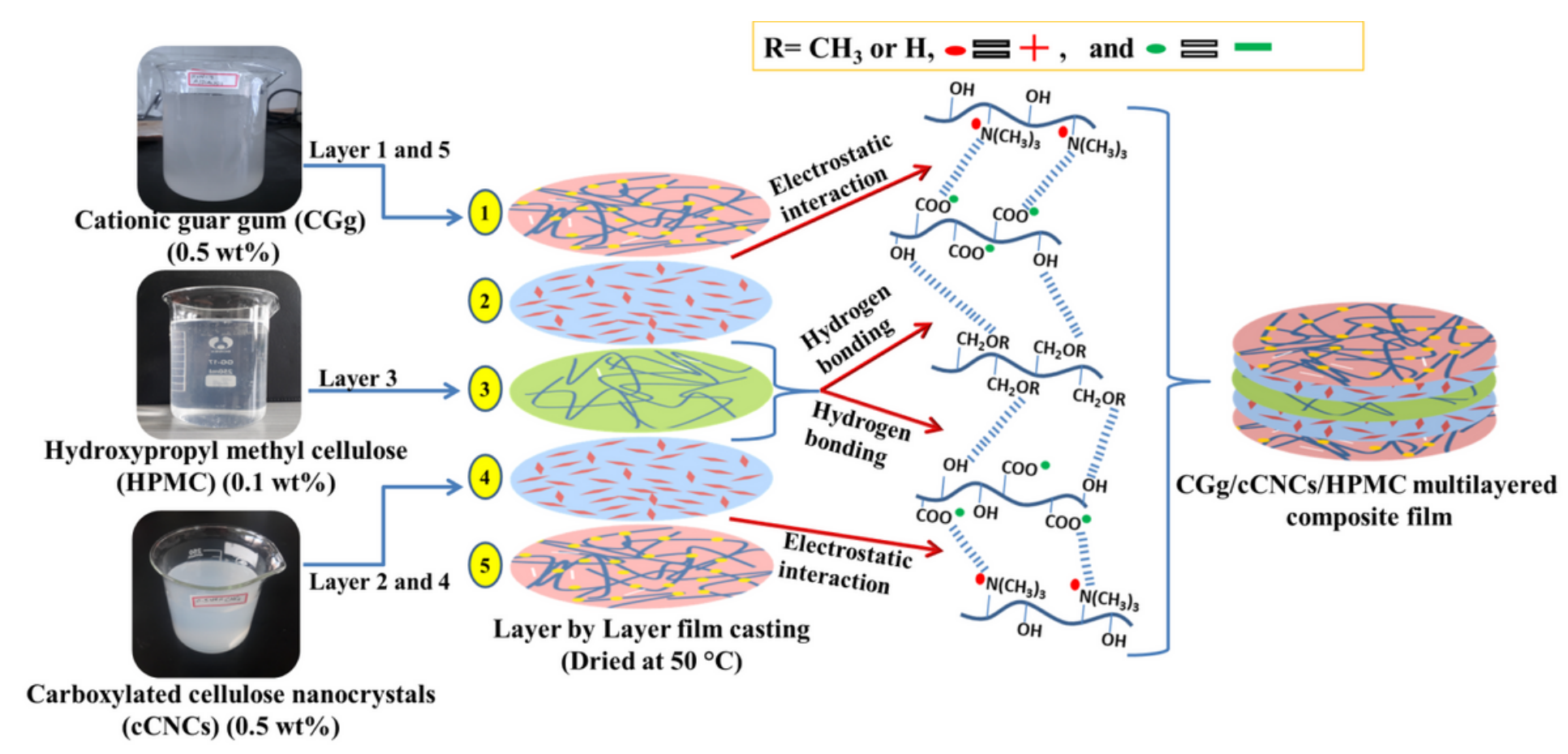

\section{Figure 1}

Schematic diagram of $\mathrm{CGg} / \mathrm{cCNCs} / \mathrm{HPMC}$ multilayered composite film fabrication by LBL self-assembly technique. 

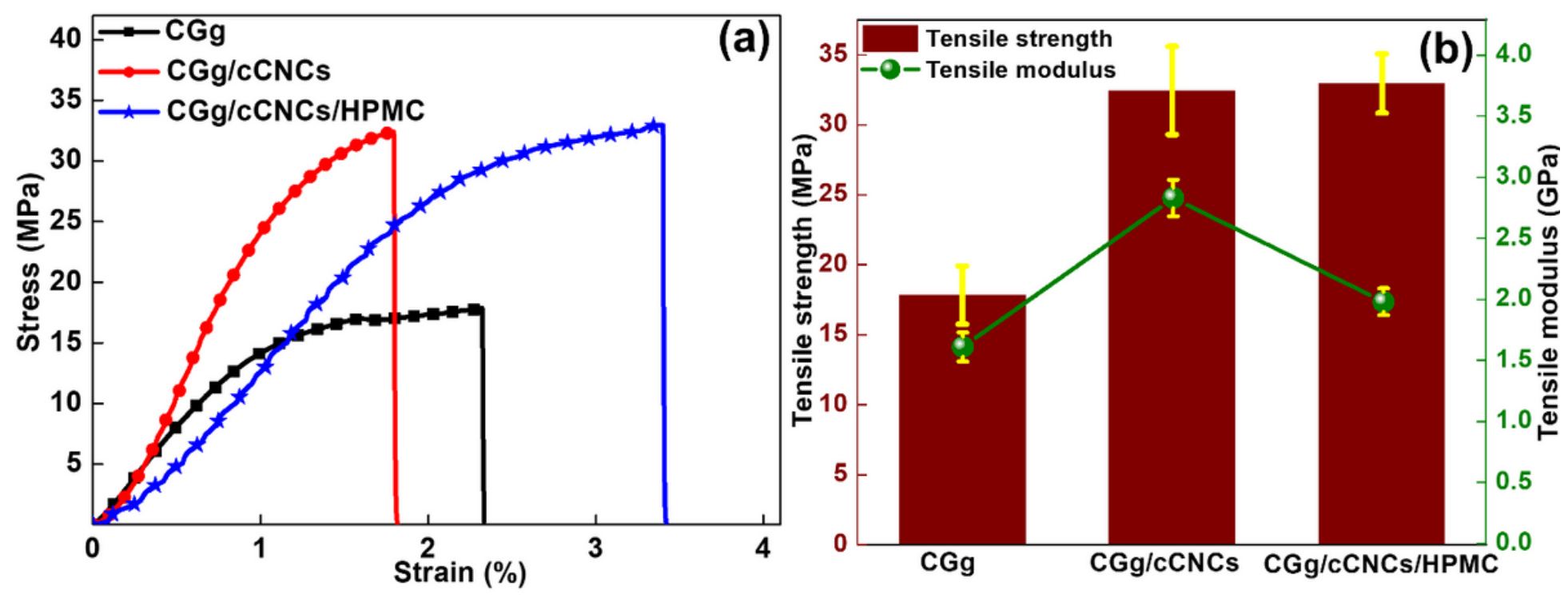

Figure 2

Mechanical properties of neat CGg and multilayered composite films (a) stress-strain curves and (b) tensile strength and modulus. 


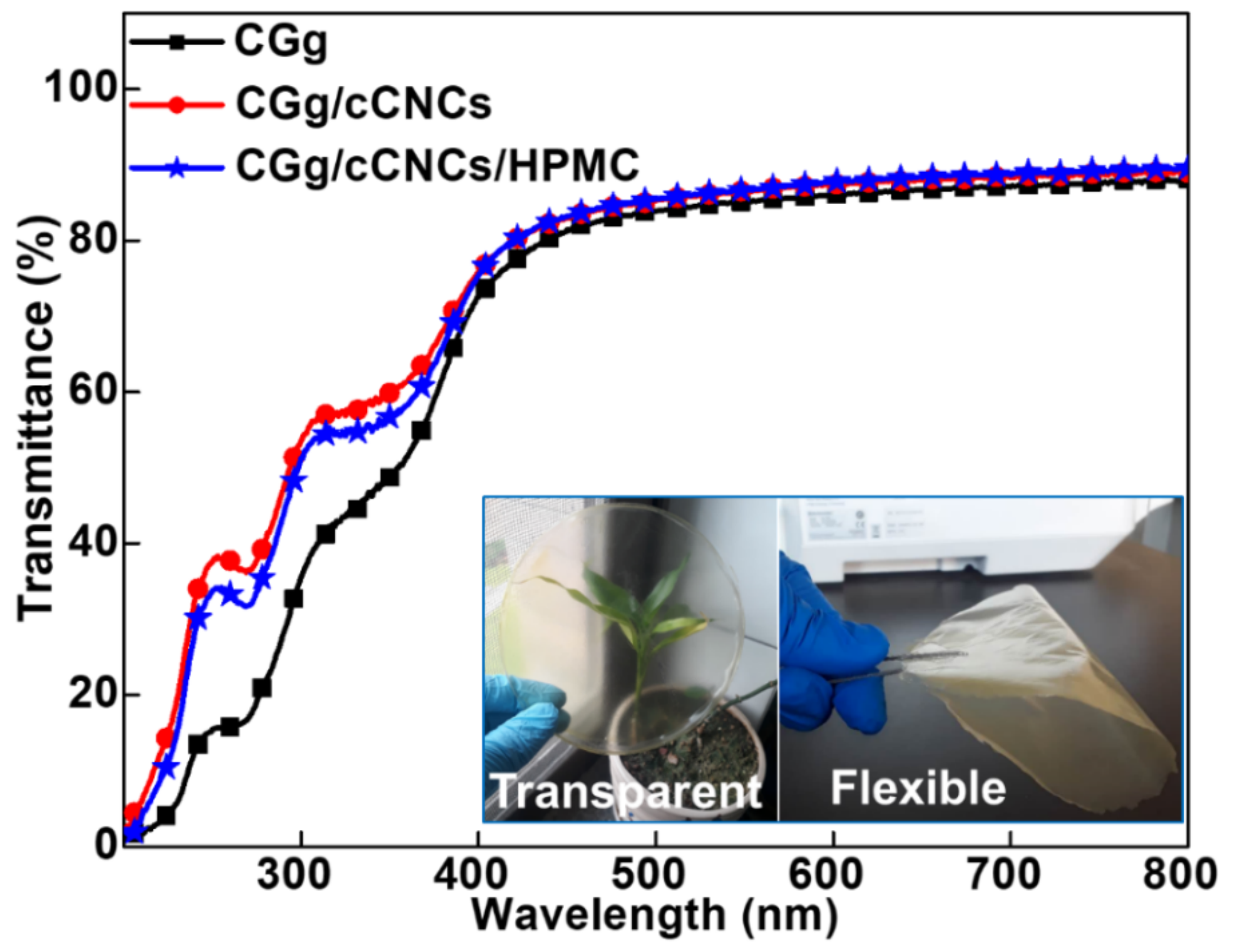

Figure 3

UV-Vis transmittance spectra of neat CGg film, and CGg/cCNCs and CGg/cCNCs/HPMC multilayered composite films. (the inset shows the photograph of $\mathrm{CGg} / \mathrm{cCNCs} / \mathrm{HPMC}$ multilayered composite film).
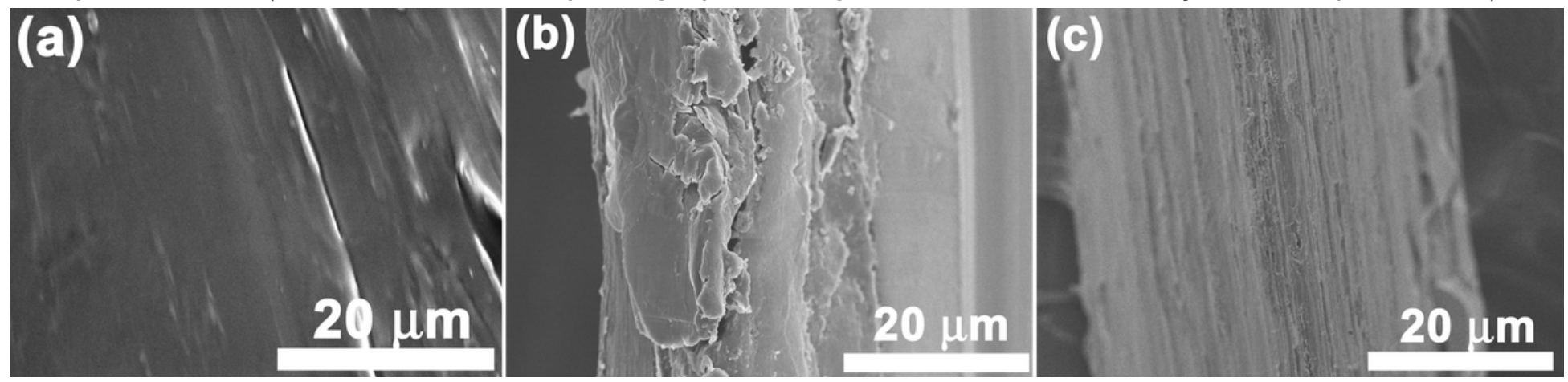

Figure 4 
FESEM images showing cross section of (a) CGg film (b) CGg/cCNCs multilayered composite film and (c) $\mathrm{CGg} / \mathrm{cCNCs} / \mathrm{HPMC}$ multilayered composite film.

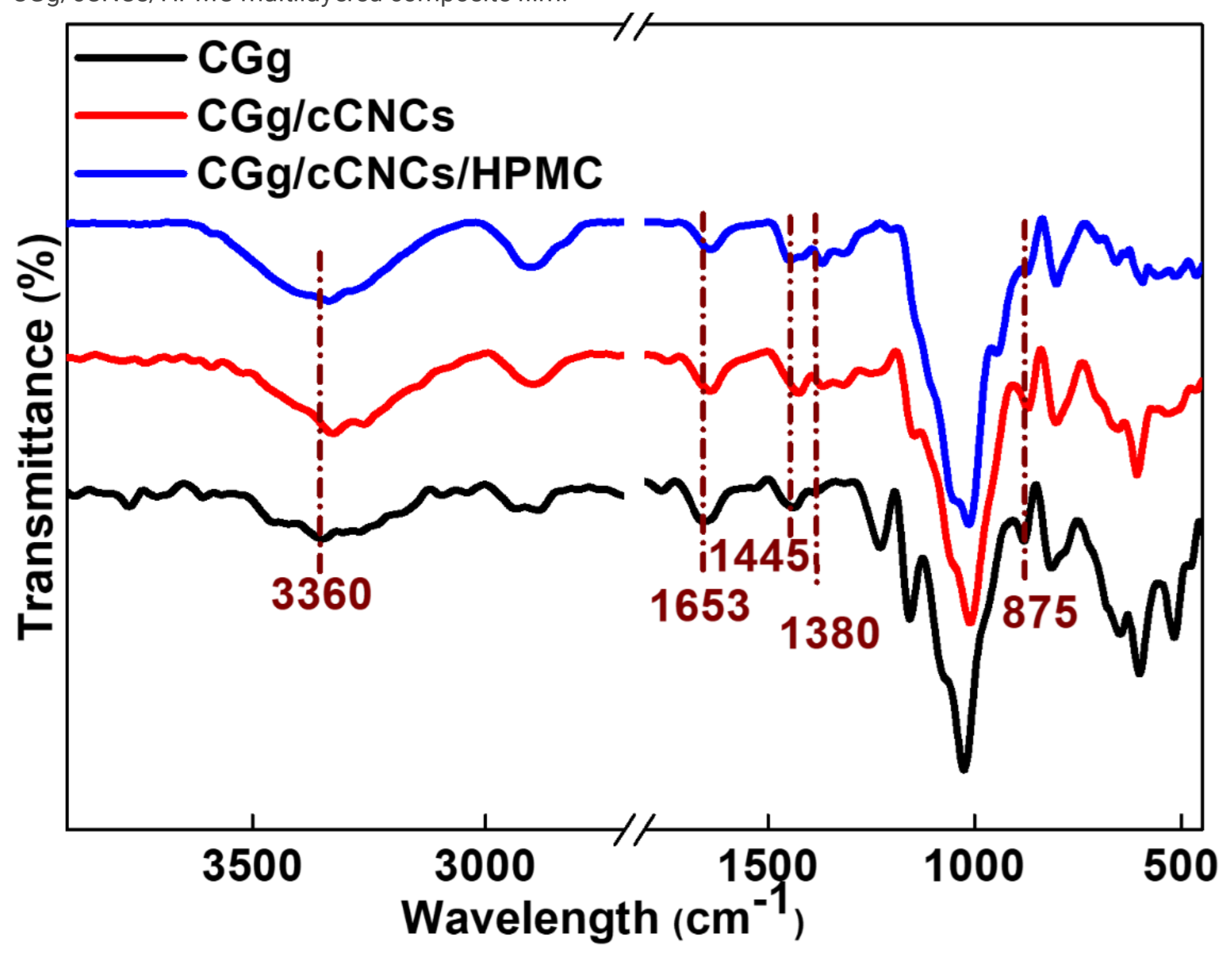

Figure 5

FTIR-ATR spectra of neat CGg film, and CGg/cCNCs and CGg/cCNCs/HPMC multilayered composite films. 


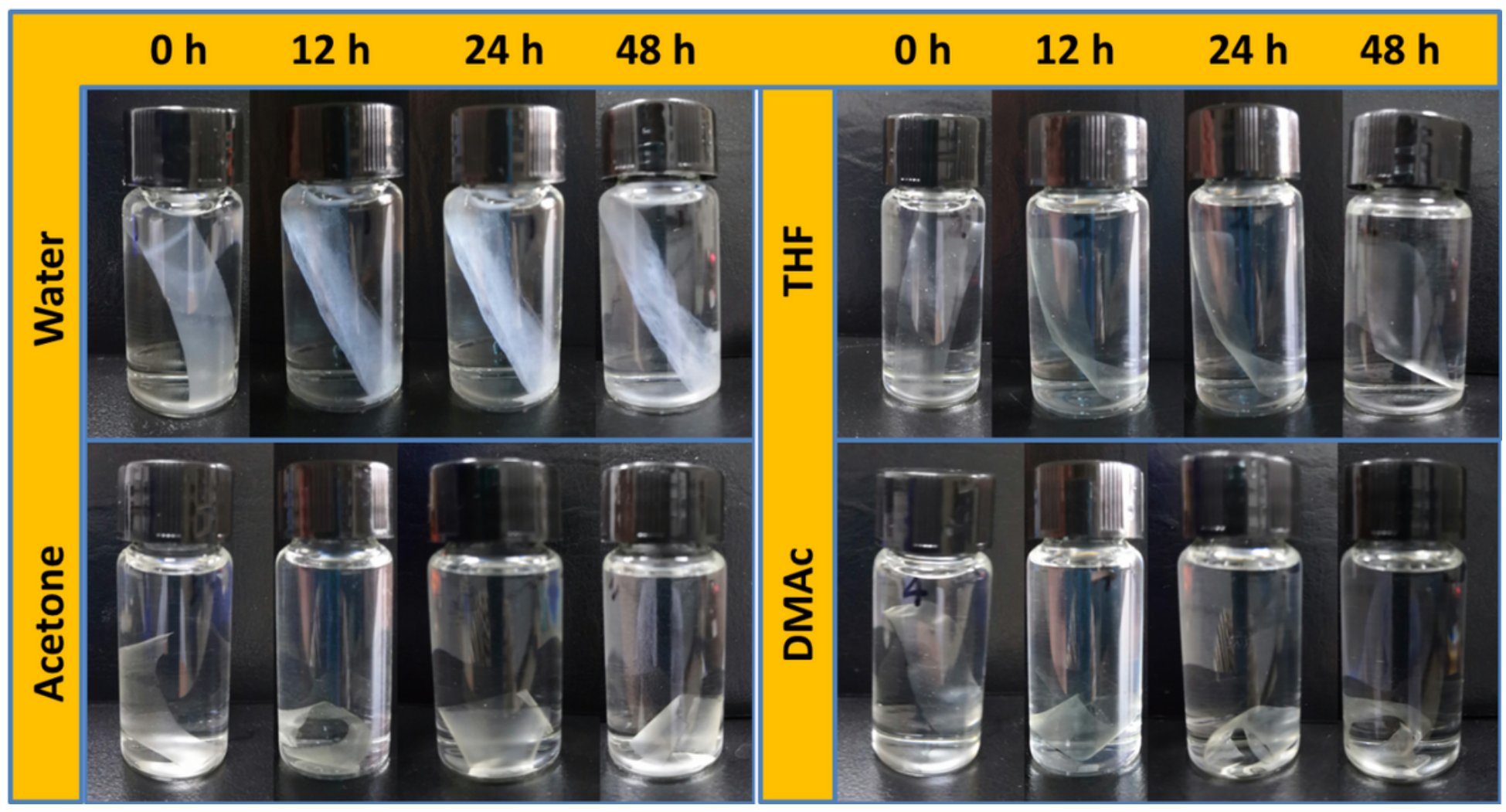

Figure 6

Photographs of solvent resistance test of $\mathrm{CGg} / \mathrm{cCNCs} / \mathrm{HPMC}$ multilayered composite film. 

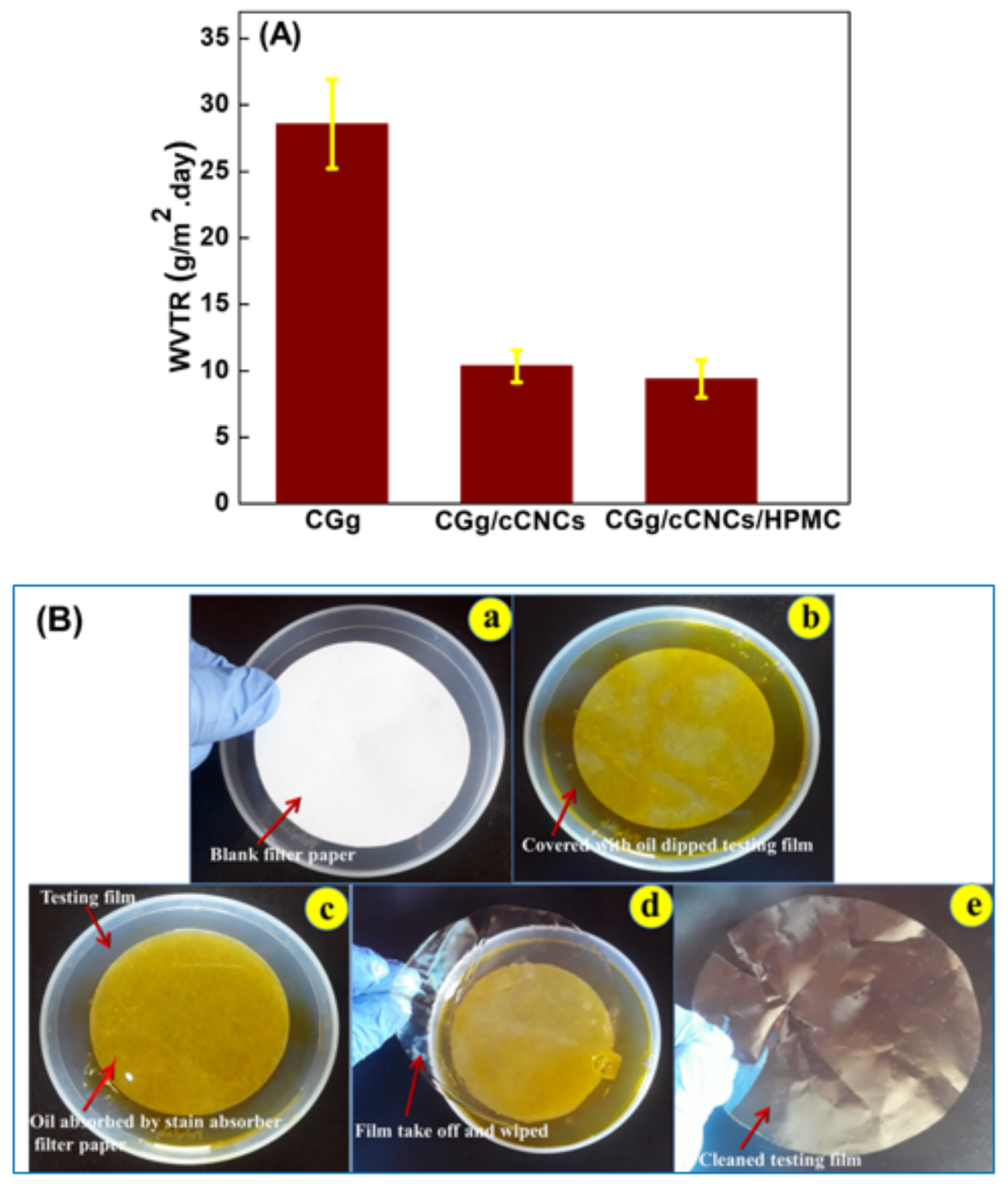

Figure 7

(A) Water vapor transmission rate of $\mathrm{CGg}$ film, and $\mathrm{CGg} / \mathrm{cCNC}$ and $\mathrm{CGg} / \mathrm{cCNCs} / \mathrm{HPMC}$ multilayered composite films. (B) Photographs for oil resistance test of $\mathrm{CGg} / \mathrm{cCNCs} / \mathrm{HPMC}$ multilayered composite film.

\section{Supplementary Files}

This is a list of supplementary files associated with this preprint. Click to download.

- Supplementarylnformation.docx 